\title{
Controlling for Unsafe Events in Dense Traffic through Autonomous Vehicles
}

\author{
Invited Talk Abstract \\ Daniel B. Work, R. Stern, \\ S. Cui, H. Pohlmann, B. Seibold \\ Temple University \\ B. Piccoli \\ F. Wu, M. Churchill \\ University of Illinois at \\ Urbana-Champaign \\ dbwork@illinois.edu \\ R. Bhadani, M. Bunting, \\ J. Sprinkle \\ M.L. Delle Monache \\ INRIA University, Grenoble \\ Rutgers University \\ University of Arizona \\ R. Haulcy \\ Yale University
}

\begin{abstract}
This talk focuses on stop-and-go instabilities in dense traffic flows, and how autonomous vehicles can be applied to control for these instabilities.

\section{CCS CONCEPTS}

- Applied computing $\rightarrow$ Transportation; $\bullet$ Computer systems organization $\rightarrow$ Embedded and cyber-physical systems; Robotic control;

KEYWORDS

Traffic flow, Sugiyama experiment

\section{ACM Reference format:}

Daniel B. Work, R. Stern, F. Wu, M. Churchill, S. Cui, H. Pohlmann, B. Seibold, B. Piccoli, R. Bhadani, M. Bunting, J. Sprinkle, M.L. Delle Monache, N. Hamilton, and R. Haulcy. 2017. Controlling for Unsafe Events in Dense Traffic through Autonomous Vehicles. In Proceedings of 1st International Workshop on Safe Control of Connected and Autonomous Vehicles, Pittsburgh, PA USA, April 2017 (SCAV'17), 1 pages.

DOI: $10.1145 / 3055378.3055380$
\end{abstract}

\section{OVERVIEW}

It was shown by Sugiyama [1] that stop and go waves will emerge from human-driven traffic even without physical bottlenecks (e.g., merging traffic or a reduced number of lanes). In this research, we describe the metrics applied to these kinds of traffic flows, and how those metrics are related to estimates of safety of the entire flow. The metrics begin with those applied to traffic, namely throughput

Permission to make digital or hard copies of all or part of this work for personal or classroom use is granted without fee provided that copies are not made or distributed for profit or commercial advantage and that copies bear this notice and the full citation on the first page. Copyrights for components of this work owned by others than ACM must be honored. Abstracting with credit is permitted. To copy otherwise, or republish, to post on servers or to redistribute to lists, requires prior specific permission and/or a fee. Request permissions from permissions@acm.org.

SCAV'17, Pittsburgh, PA USA

(C) 2017 ACM. 978-1-4503-4976-5/17/04 ..\$15.00

DOI: $10.1145 / 3055378.3055380$ (number of vehicles per hour), and instantaneous average velocity of all vehicles in the flow. We further define new metrics that may be applied to specific safety concerns such as following distance, by measuring how often a driver brakes, and with what magnitude.

In order to control the traffic flow according to these metrics, we must explore how to perform in-situ measurements by various sensors availble both in the flow and external to vehicles. Once we have established the baseline for metrics, we explore how to measure individual vehicles' trajectories and velocities with various on-board and environmental sensors. These include the use of OBDII sensors installed in each vehicle in the flow, as well as external cameras and on-board distance sensors. Since these sensors use different modalities with various rates and time horizons, we also discuss how to validate measurements and synchronize them with respect to common events.

Methods for control are discussed based on the available data and their reliability. Results from simulation and their limitations factor in to how the models applied in simulation must be compared to models observed in physical experiments. The talk concludes with discussion of results from these physical experiments that have drivers with full-sized cars replicate the scenario of the Sugiyama experiment, but with velocity control implemented with only one of the vehicles.

\section{ACKNOWLEDGMENTS}

This work supported by the National Science Foundation project "Control of Vehicular Traffic Flow via Low Density Autonomous Vehicles" under awards CNS-1446435, 1446690, 1446702, 1446715.

Any opinions, findings, and conclusions or recommendations expressed in this material are those of the author(s) and do not necessarily reflect the views of the National Science Foundation.

\section{REFERENCES}

[1] Yuki Sugiyama, Minoru Fukui, Macoto Kikuchi, Katsuya Hasebe, Akihiro Nakayama, Katsuhiro Nishinari, Shin ichi Tadaki, and Satoshi Yukawa. 2008. Traffic jams without bottlenecks-experimental evidence for the physical mechanism of the formation of a jam. New fournal of Physics 10, 3 (2008), 033001. http://stacks.iop.org/1367-2630/10/i=3/a=033001 